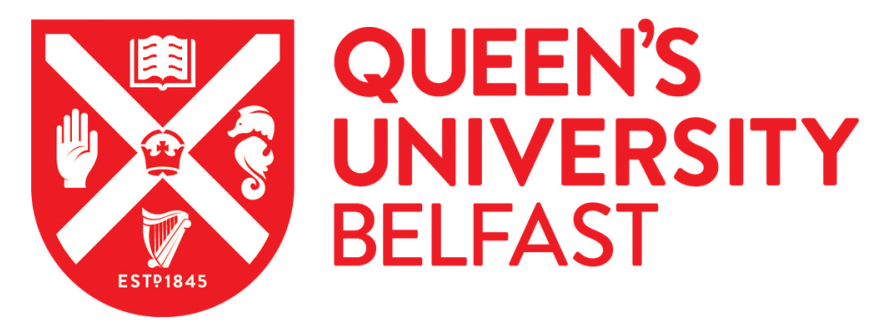

\title{
Molecular Profiling of RNA Tumors Using High-Throughput RNA Sequencing: From Raw Data to Systems Level Analyses
}

da Silveira, W. A., Hazard, E. S., Chung, D., \& Hardiman, G. (2019). Molecular Profiling of RNA Tumors Using High-Throughput RNA Sequencing: From Raw Data to Systems Level Analyses. Methods in Molecular Biology, 1908, 185-204. https://doi.org/10.1007/978-1-4939-9004-7_13

Published in:

Methods in Molecular Biology

Document Version:

Peer reviewed version

Queen's University Belfast - Research Portal:

Link to publication record in Queen's University Belfast Research Portal

Publisher rights

(C) 2019 Springer Nature Switzerland AG.

This work is made available online in accordance with the publisher's policies. Please refer to any applicable terms of use of the publisher.

\section{General rights}

Copyright for the publications made accessible via the Queen's University Belfast Research Portal is retained by the author(s) and / or other copyright owners and it is a condition of accessing these publications that users recognise and abide by the legal requirements associated with these rights.

Take down policy

The Research Portal is Queen's institutional repository that provides access to Queen's research output. Every effort has been made to ensure that content in the Research Portal does not infringe any person's rights, or applicable UK laws. If you discover content in the Research Portal that you believe breaches copyright or violates any law, please contact openaccess@qub.ac.uk. 

Systems Level Analyses

Willian A. da Silveira, E. Starr Hazard, Dongjun Chung, and Gary Hardiman

4

Abstract

RNAseq is a powerful technique enabling global profiles of transcriptomes in healthy and diseased states. In this chapter we review pipelines to analyze the data generated by sequencing RNA, from raw data to a system level analysis. We first give an overview of workflow to generate mapped reads from FASTQ files, including quality control of FASTQ, filtering and trimming of reads, and alignment of reads to a genome.

Then, we compare and contrast three popular options to determine differentially expressed (DE) transcripts (The Tuxedo Pipeline, DESeq2, and Limma/voom). Finally, we examine four tool sets to extrapolate biological meaning from the list of DE genes (Genecards, The Human Protein Atlas, GSEA, and ToppGene). We emphasize the need to ask a concise scientific question and to clearly under stand the strengths and limitations of the methods.

Key words: High-throughput sequencing (HTS), RNAsequencing (RNAseq), FASTQ, Tuxedo pipeline, HTSeq, DESeq 2, Limma/Voom, Gene Set Enrichment Analysis/GSEA, TOPPGENE

\section{Introduction}

Classical techniques in genetics and molecular biology remain the gold standard when one wants to detect the presence and sequence of a gene (i.e., using polymerase chain reaction (PCR) and Sanger sequencing techniques), its mRNA expression level (i.e., using quantitative PCR (qPCR)), and the corresponding protein levels (i.e., using western blots) [1]. The human genome encodes approximately 25,000 genes, with thousands of them expressed in multiple combinations in diverse cellular contexts. Furthermore multiple isoforms exist for the same gene with many possible downstream post-translation modifications [2]. The emergence and application of high throughput approaches in the past decade, the so-called "omics" fields have ignited a revolution in biological research. Classical genomics techniques only allow investigation of a small number of genes and proteins at the same time, where as "omics" approaches (including high throughput RNA sequencing) enable investigation of the entire mRNA content at the same time $[1,2]$.

Transcriptomics is the study of the transcriptome, i.e., the complete set of RNA transcripts that are produced by the genome, under specific circumstances or in a specific cell, using highthroughput methods, such as RNAseq [3]. Comparison of transcriptomes allows the identification of genes that are differentially expressed in distinct cell populations, for example in healthy or tumor tissues, or in response to therapeutic regimes. In this chapter we discuss the methods to analyze and interpret data from human tumor samples generated by RNAseq technology. We cover the key steps and the progression from the "FASTQ" files generated by the sequencing instrument through a list of differentially expressed genes, and system level analyses.

Although the methods and workflows described in this chapter are best suited to a Linux environment, there are options to run many of these programs in Windows or Mac OS X as well, e.g., using Cygwin (https://www.cygwin.com/) in Windows. A basic knowledge of the command line environment, file structures and rudimentary coding skills are assumed. More details about these 
programs and the statistical models underlying them used can be found in the references and/or in the links of the websites in the Materials section. As noted by Mayer-Scho"nberger and Cukier, "the data can reveal secrets to those with the humility, the willingness, and the tools to listen" [4].

\section{Materials}

\subsection{Computing Infrastructure}

There are many challenges associated with selecting and implementing the right set of tools. Bioinformatics analyses are complex, multistep processes composed of multiple software applications. Ideally, many of the programs used for RNAseq analysis are designed to show the optimal performance in a high-performance computing environment. However, these programs can still run in a sufficiently powerful and well-configured laptop or desktop machine, e.g., an Intel Core i7 processor with storage RAID 0 configured 2 parallel 1-TB hard disk drives. We recommend at least $4 \mathrm{~GB}$ of RAM, preferably $8 \mathrm{~GB}$.

\subsection{Software Tools and Genome Build(s)}

SRA Toolkit 2.9.0 (https://github.com/ncbi/sra-tools/wiki/Downloads, http://www.ncbi.nlm.nih.gov/books/NBK158900/), Windows/Linux/MAC OS X.

FastQC v0.11.7 (http://www.bioinformatics.babraham.ac.uk/projects/FASTQc/), Windows/Linux/MAC OS $X$.

FASTX-Toolkit (http://hannonlab.cshl.edu/fastx toolkit/), Linux/MAC OS X/Web based.

Cutadapt (https://cutadapt.readthedocs.io), Python Language. Windows/Linux/MAC OS X.

Bowtie1 for reads between 35 and 50 bp (http://bowtie-bio.sourceforge.net/manual.shtml). LinuX/MAC OS X.

Bowtie2 for reads greater than $50 \mathrm{bp}$ (http://bowtie-bio.sourceforge.net/bowtie2/manual.shtml). Linux/MAC OS $X$.

SAMtools (http://samtools. sourceforge.net/) . Windows/Linux/MAC OS X. Samtools also have an "R" compatible version "Rsamtools," available from the Bioconductor website.

Human Reference Genome Sequence, hg38 (http://hgdownload.cse.ucsc.edu/downloads.html).

TopHat (https://ccb.jhu.edu/software/tophat/manual.shtml) ). Linux/MAC OS X.

Cufflinks package (Cufflinks, CuffMerge, CuffMerge, Cuffdiff)(http://cole-trapnelllab.github.io/cufflinks/tools/). Linux/MAC OS X.

CummeRBound (https://bioconductor.org/packages/release/bioc/html/cummeRbund.html). R Language. Windows/Linux/MAC OS X.

HTSeq (http://www-huber.embl.de/HTSeq/). Python Language. Windows/Linux/MAC OS X. 
Comprehensive gene annotation (GRCh38.p7, ".GTF file").

(http://www.gencodegenes.org/releases/25.html).

DESeq2 (https://bioconductor.org/packages/release/bioc/html/DESeq2.html). R Language.

Windows/Linux/MAC OS X.

Limma/Voom (https://bioconductor.org/packages/release/bioc/html/limma.html). R Language. Windows/Linux/MACOS X.

Venny (https://www.stefanjol.nl/venny) Web tool.

ToppFun (https://toppgene.cchmc.org/) Web tool.

GSEA tool and Website (http://software.broadinstitute.org/gsea/index.jsp). Web tool and a program compatible with Windows/Linux/MAC OS X.

\section{Methods}

A schematic of data analysis pipelines is presented in Fig. 1. To initiate the analyses, it is necessary to have the FASTQ files that contain the information of sequenced reads and the quality score for each nucleotide. These FASTQ files can be obtained directly from the sequencing machine, as in the case depicted in the flowchart, or from the Sequence Read Archive (SRA) repository (https://www.ncbi.nlm.nih.gov/sra), where it is required to convert the downloaded ".SRA" file to FASTQ using the SRA Tool kit. Then the quality of the FASTQ file can be checked using the FastQC program and the results from it can be used as parameters in the "FASTX" Tool Kit and/or in Cutadapt to perform trimming and filtering of the reads. Then, the FASTQ file is now ready for the alignment of reads to the genome, which can be attained using the TopHat program with a reference genome, or any other aligner tool. At this point our pipeline bifurcates, the list of DE genes can be obtained using the Tuxedo pipeline (comprised by Cufflinks, Cuffmerge and Cuffdiff) using FPKM (Fragments Per Kilobase of transcript per Million mapped reads). Alternatively, the list of DE genes can also be obtained using the counts of the reads coming from the HTseq program and then processed by DESEQ2 or Limma/Voom. The biological meaning of the DE gene list can be analyzed gene by gene using "The Human Protein Atlas" and "Genecards" websites or at a system level using the GSEA and/or ToppGene Suite. We discuss each step more in detail below.

\subsection{From FASTQ Files to BAM/SAM Files}

\subsubsection{Raw Data-FASTQ Files}

A typical RNAseq data analysis begins with FASTQ files. There is a single FASTQ that corresponds to each RNA sample sequenced. If the study is paired-end sequencing, there are two FASTQ files, each of which corresponds to the left and right reads of each DNA fragment [5]. In the early days of high throughput sequencing, sequenced reads were often stored in the FASTA file format, which is a text file containing a sequence of nucleic acids or amino acids. The FASTQ file is an extension of this file format, i.e., a FASTA that contains both a sequence of nucleic acids and a quality score for that particular sequence. The quality score provides a measure of confidence in the 
sequencing data [6]. These files are typically large and easily reaching tens to hundreds of gigabytes in size.

In the FASTQ file, each raw sequence is described with four lines (Fig. 2a). The first line begins with an "@” and contains an identifier for the sequence. The second line contains the raw sequence itself, as found in the FASTA file. The third line starts with a " + " and optionally repeats the content of the first line. The fourth line contains the phred quality score $(Q)$, a measure of the quality of the identification of the base (Fig. 2b) [6]. Phred was originally developed as a quality score for Sanger sequencing data and was adapted in FASTQ. This quality score is calculated by comparing chemical parameters of the given sequencing process with the parameters of a large dataset of known accuracy $[6,7]$.

In this review, we are using the dataset GSE81167, available from the Gene Expression Omnibus (GEO), for the purpose of illustration. This study evaluated the impact of ZEB1 expression in HCC827 cells, which are human lung cancer cell lines [8]. ZEB1 is one of the principal transcription factors involved in the epithelial-to-mesenchymal transition, a key event in tissue invasion and metastasis [9]. This dataset is available for GEO in the short read archive ".SRA" file format. We used the FASTQ-dump command line program from the SRA Toolkit 2.9.0 to convert files from ".SRA" to ".FASTQ". In most cases, SRA files downloaded from repositories, as from the Gene Expression Omnibus, can be directly converted to SAM files (Sequence Alignment/Map Format), affording savings to both the user's time and central processing unit (CPU) cycles. For didactic reasons, we start from the FASTQ files.

\subsubsection{Quality Control of FASTQ Files with FastQC}

Although the FASTQ file provides the quality score for each base in every sequence, checking it manually is time-consuming and impractical. FastQC is a program that allows evaluation of the quality of the FASTQ file as a whole. The program uses the FASTQ file as input, and yields three types of FastQC files: A report in the ".html" format, the same report in the compressed ".zip" file, and the "FastQC" folder with the unzipped version of the files [10]. As presented in Fig. 3, the report analyzes a number of items, basic statistics, per-base sequence quality, and other metrics including sequence content, GC content, sequence duplication and the presence of adapter and overrepresented sequences. In our example, even in what can be considered a good report (Fig. Upper Panel), per base sequence content and K-mer content are flagged as potentially problematic. The per-base sequence content suggests sequences with GC-content are over-represented, which might potentially imply contamination from ribosomal RNA (rRNA). K-mer bias occurs when overrepresented sequences result in the K-mers derived from these sequences being highly enriched [10]. In our bad report (Fig. 3 Lower Panel), in addition to problems with the quality of the nucleotides sequenced that occur as the read length grows, we have a warning for per tile sequence quality, sequence duplication level and over-represented sequences and a failure for per base sequence content, per sequence GC content and again the K-mer content. Both the warnings and the failures indicate a problem at the library preparation step [10].

\subsubsection{Preprocessing-Filtering and Trimming}


Once we have the information on sample quality, the next step is to filter out bases and reads with low quality and to extract adaptor, primer, and poly-A tails from the data. To do that, we use the "fastx_trimer" and "fastq_quality_trimmer" programs to filter the adapters, poly-A tails, and PCR primer sequences and "fastq_quality_filter" to filter out low quality bases and/or reads. These programs are a part of the FASTX-Toolkit [11].

By taking the FASTQ files as input, a sequential use of the two FASTX-Toolkit programs, "fastx_trimer" and "fastq_quality_trimmer", will return a "cleaned" FASTQ file. FASTX-Toolkit can be used only in the command line, which requires some basic knowledge of Linux. The information acquired with the FastQC program must be used in the parameters of the "fastq_quality_filter", when necessary.

Alternatively, we can use the program "Cutadapt" [12] for trimming adapter sequences, primers, and poly-A tails from the FASTQ file. Cutadapt accepts the FASTQ as input and operates in any system that runs Python. However the program limits itself to the file cleaning and does not support base quality filtering [12].

\subsubsection{Preprocessing - Read Alignment to the Genome}

We now have good quality reads and a high level of confidence that the original RNA sequence is represented in the FASTQ file. Is it necessary now to align the reads to our reference genome, in this case the Human Genome, version hg38 [13]. We use, TopHat, a part of the Tuxedo pipeline, described by Trapnell and collaborators in 2012 [14]. TopHat is integrated with the Bowtie and SAMtools programs, uses the FASTQ files as input, uses the selected genome as the reference, and generates a SAM file as output. It runs only in the linux shell $[14,15]$. To generate the alignment, firstly TopHat uses the Bowtie program to align the reads to the genome. Bowtie is a fast and efficient short read aligner, but unfortunately cannot align reads with large gaps compared with the reference, which makes it unsuitable to align reads that span introns or fusion genes. After the first round of alignment, TopHat breaks the unmapped reads into smaller parts and run a new alignment round using Bowtie. This is one of the key strengths of TopHat, because it permits the identification of splicing variants and fusion genes [14, 15].

The output generate a folder with a number of files and the most important of them are: the "align_summary.txt" with a summary of the alignment, "accepted_hit.bam" with a list of read alignments in the SAM format (Fig. 4), (".bam" is a "binary SAM"), "unmapped.bam" with the information on the reads that could not be aligned, "deletion.bed", "insertion.bed", and "juntions.bed", with the information described in its name in the Browser Extensible Data (BED) format.

\subsection{From BAM/SAM Files to a List of Differentially Expressed Genes}

The need to analyze RNAseq data has given rise to a plethora of methods, with different characteristics and assumptions [16]. Here we describe three widely used tools, the Tuxedo pipeline, DESeq2 and Limma/voom [14, 17-19]. Tuxedo employs FPKM (Fragments Per Kilobase Of Exon Per Million Fragments Mapped) in its analysis pipeline [14], while DESeq2 and Limma/voom accept the 
counts derived from HTseq analysis as Input $[16,20]$. As both HTseq and Cufflinks accept the ".BAM" files, we can use the "accepted_hit.bam" from the previous step [14, 15] as input for these programs.

\subsubsection{The TUXEDO Pipeline}

The TUXEDO package consists of Cufflinks, Cuffmerge, and Cuffidff programs, more detailed descriptions of these methods are available in the supplementary data of Trapnell et al. [21]. First, the Cufflinks program that uses the ".bam" files from TopHat to assemble the reads into the most probable transcripts and will give a ".gtf" file with the FPKM for the transcripts. Then Cuffmerge takes the ".gtf" files of all the samples and generates a merged ".gtf" file as output. Cuffdiff estimates the differential expression not only of the genes but also of their isoforms and the promoters used (TSS - transcription start site) [14, 21]. The output is tab-delimited text files, including the ".diff" files with the results and other files with information about the analyses, shown in part in Fig. 5. The pipeline also contains the program CummeRBund that can be optionally used to manage, integrate, and visualize the results produced by the Cufflinks package [14].

The Tuxedo Pipeline was for many years the de facto RNAseq analysis pipeline, due to its attractive ability to provide gene expression, isoform variation, and TSS use. Nonetheless, it can only be executed in the Linux command line, has multiple command steps, and require expertise in this environment. Furthermore, it has also been reported that the Tuxedo pipeline has a lower precision and sensitivity than DESeq 2 and Limma/Voom [16, 17].

\subsubsection{HTSeq}

HTSeq uses ".bam" files from the samples and a ".gtf" or ".gff" file with the gene models as input. It is scripted in Python and the "HTSeq-count" script is able to count how many aligned reads overlap with the exons of the genes, not considering differential splicing. Reads that align with more than one gene are discarded, excluding fusion genes. The output is a tab-delimited text ".txt" file with two columns: one with the gene name and one with the counts (Fig. 6) [20].

The "count" value is exactly as described. It is a measure of how many reads for that gene exist in the ".bam" files. For the nature of the analysis, the "count" is nonnegative integer valued. A read can be counted as belonging to a gene ( +1 in the "count" value) or not [20]. This value is not corrected or normalized in any way by HTSeq and downstream analysis will have to address this issue. At this point, it is interesting to note that the same sample sequenced with different depths will have different count values for each gene simply because of the different quantity of reads generated by the sequencer. This issue needs to be considered when working with samples from different origins and/or from multiple datasets [20,22]. It is possible to introduce a prefiltering step at this point, i.e., excluding the genes that have zero counts in all samples, to reduce the needed computation time and improve the statistical power as this step can affect the false discovery rate control [23].

\subsubsection{DESeq2}


246 The DESeq2 package uses unnormalized count data, such as that provided from HTseq, as input. The program internally corrects for library size, so raw counts needs to be used in order to run the analyses [24]. To run the analyses, it is necessary to create a DESeqDataSet object, using the ".txt" counts files and an user-specified design matrix, i.e., assignment of samples to different treatment groups [24]. At this point, the "DESeq" function is invoked to perform the differential analysis. The output is a table containing the gene symbol, the base 2 log-transformed fold change, the pvalue, and the adjusted $p$-value (or q-value, a measure of false discovery) (Fig. 7). DESeq2 assumes a negative binomial linear model to describe over-dispersed count data from RNAseq data. It uses an empirical Bayes method for more robust and accurate estimation of parameters for dispersion and fold change, by taking into account small numbers of replicates and low read counts in RNAseq data. Finally, DESeq2 uses the Wald test to estimate significance of differential expression, and the Benjamini-Hochberg correction to control the false discovery rate $[17,24]$.

\subsubsection{Limma/Voom}

260

Limma is one of the well-known R packages used for differential gene expression analysis. It was initially developed for microarray analyses, prior to the emergence of RNAseq, and has been updated to facilitate analysis of RNASeq data [25]. Limma uses linear models to specify the experimental design, empirical Bayes method to moderate the standard errors between genes, and uses the t-test to calculate the differential expression $\mathrm{p}$-value, while providing multiple choices for adjustment of the $p$-value for multiple testing, including the Benjamini-Hochberg $[19,25]$. The use of Limma is well suited for small numbers of samples per group, as few as two, and also powerful when used in multifactor designed tests $[16,25]$. It has often reported that DESeq2 and Limma have similar precision and sensitivity in their analysis results $[16,17]$.

As with DESeq2, Limma accepts the ".txt" count files from HTseq as input but internally transforms the data because Limma assumes the t-distribution for gene expression values as it was originally developed for microarray datasets $[25,26]$. In order to address this issue, Limma uses the Voom transformation, i.e., log transformation of counts per million (cpm) with associate precision weights [18]. The Voom transformation is the key step in this analysis, as its log transformation helps gene expression values satisfy the $t$-distribution assumption of Limma, while using cpm instead of raw count normalizes gene expression values across replicates $[18,25]$. Once we have the transformed table, we use the "ImFit" function to fit the data to a linear model, which informs the design of the experiment as in the case of DESeq2. The next step is to call the "eBayes" function that will calculate the moderated $t$-statistics and log-odds of the differential expression using an empirical Bayes moderation. The output is a table containing the gene symbol, the fold change in log scale with base 2 , the $p$-value, and the adjusted p-value as shown in Fig. 8. 
vary from tens to thousands genes. In this chapter we consider the analysis comparing two groups of experimental conditions but this can also be easily extended to multiple groups as well. The simplest way to compare the genes from different experimental conditions is using Venn diagrams, which permits to assess what is in common and what is unique in the gene lists of your differential expression analysis [27]. In addition, when only a handful of genes are of interest, deeper interrogation of each gene can be also implemented using Genecards and The Human Protein Atlas $[28,29]$. Nonetheless, most of the time, the differentially expressed gene list might be too long for such gene-by-gene analyses and as a result, system level analyses might often be more appropriate, for example, by using GSEA and/or Toppfun [30, 31].

\subsubsection{Venn Diagrams - Venny}

Area-proportional Venn diagrams are a useful graphic approach to compare different analyses. BioVenn is a convenient web application for the comparison and visualization of biological lists [27]. For example, we can visualize how the gene lists generated by different $D E$ analysis programs described above are related to each other (Fig. 9). On the other hand, Venn diagrams can also be used to compare the results of two different comparisons between groups and/or experimental conditions. As we can see, the way we analyze our data influence the results. If information on one specific gene being differentially expressed in a given condition is needed, it is often necessary to validate it with qPCR [1].

\subsubsection{Genecards and the Human Protein Atlas}

Once in possession of a list of ranked DE genes, the next task is to make sense of it in a biological context. One possibility is to check the DE gene list on a gene by gene basis, or at least the top ranked genes in the list, for their function and expression in different tissues. For this step there are two web services that can be utilized: Genecards, a repository with information of gene and protein function, expression and known interactions [28] (Fig. 10); and The Human Protein Atlas, with information on RNA and protein levels in different tissue and cancer types, and immunostaining data from images derived from tissue histology [29] (Fig. 11). Although this method is useful to analyze a small list of genes, or the function of the top ranked genes in a list, it is unpractical when we have a list of hundreds or thousands of DE genes. In this case, a system level analysis is required.

\subsubsection{Gene Set Enrichment Analysis (GSEA)}

One of the key assumptions of the gene set enrichment analysis is that a moderate increase in expression of a large number of genes encoding members of a pathway may dramatically alter the flux through the pathway and may be more important than a huge increase in a single gene from that pathway [30, 32]. GSEA is available as online and desktop versions. Both use the same molecular signature database, comprised of eight collections: The Hallmark Gene sets $(H)$, the Positional gene set (C1), the Curated gene sets (C2), the Motif gene sets (C3), the Computational 
gene sets (C4), the Gene Ontology gene sets (C5), The Oncogenic signatures (C6), and the Immunologic signatures (C7) [30, 32]. The desktop version accepts a ".txt" tab-delimited table as input, although the Limma/voom transformed table is preferable, as the program was originally designed to analyze log2 values from microarray data. Count values obtained directly from HTseq are also accepted as input, although in this case it is important to check if the data needs to be normalized [33]. In the basic analyses, the samples in the table are separated into two groups, test group against control group. GSEA determines the enrichment of gene sets using a modified Kolmogorov-Smirnov test. Specifically, it calculates the Enrichment Score (ES) taking into consideration if a gene set is over represented at the top or at bottom of the ranked list, and it estimates the $p$-values using empirical phenotype-based permutation test procedure, along with their FDR $[30,33]$. The output contains the table of gene sets that are positively and negatively correlated with the groups, with ES, p values, and FDR values. In addition, GSEA also provides a graphical representation of the analysis, containing a heatmap comparing the two groups in the context of the gene set and an enrichment plot (Fig. 12). We note that in the GSEA analysis, it is important to provide the complete transcriptome as a whole as input, rather than a previously selected list of genes (e.g., from Limma/voom). The desktop version also accepts a preranked list of genes as input for the analyses. In contrast, the online version only accepts a list of previously selected genes as input, and tests the enrichment of gene sets using the hypergeometric test. Specifically, it evaluates if any of the gene sets are over-represented in the provided gene list, and generates a downloadable table with the statistically significant gene sets, the $p$-value, and the overlap between the gene sets and the provided gene list (Fig. 13).

\subsubsection{ToppGene Suite}

ToppGene suite is a web-based service, with four functionalities: ToppFun, ToppGene, ToppNet, and ToppGenet [31]. ToppFun (1), similar to the GSEA web based tool, accepts a gene list as input and provides a downloadable table with the enriched pathways as output. Additionally it also generates tables for 14 annotation categories including GO terms, pathways, protein-protein interaction, microRNAs, and related diseases [31] (Fig. 14). ToppGene (2) takes two gene lists as input, i.e., "training gene set" and "test gene set." In our case, the "test gene set" is the DE gene list while the "training gene set" is a gene list containing geneof our interest. Given these gene lists, ToppGene will run a ToppFun analyses of the "training gene set" to identify the most notable characteristics of this list, and rank the genes in the "test gene set" according to these characteristics. For example, if the "training gene set" is formed by the genes involved in Angiogenesis, the test genes will be ranked based on their relevance to Angiogenesis. Similarly, if the "training gene set" is formed by membrane proteins, the test genes will be ranked based on the degree they are related, or directly interact, with membrane proteins. ToppGene uses all 14 annotation categories to analyze the "training gene set" and rank the "test gene set" based on all of these 14 annotation categories [31].

ToppNet (3) and ToppGenet (4) compare "training gene set" with "test gene set" and construct networks of interactions. Specficially, ToppNet is based only on protein-protein interaction (PPI), while ToppGenet uses both PPI and the genes in the neighborhood in order to take in account possible indirect interaction. 
374 In this chapter, we described how to analyze RNAseq from raw data to a list of genes and related 375 systems and pathways. RNAseq has gained popularity with the emergence of high-throughput sequencing (HTS) or next-generation sequencing (NGS). These advances in conjunction with improvements in Proteomics and Metabolomics techniques and related analytical methods have introduced a paradigm shift in biomedical research. This big data landscape was unimaginable just a

379 few short years ago [34].

This postgenomics era has enabled a new way of thinking, as it becomes clearer and clearer that the events occurring in cells and tissues are much more complex than the sum of their parts [35]. The vast amount of data, from diverse origins, and the need for integration can be overwhelming. Thus it is imperative that the researcher have a clear idea of the question that is being asked, the data and concepts that are used to formulate a hypothesis, and the assumptions that statistical methods are based on, and the inherent limitations of these methods.

\section{Acknowledgments}

This work was conducted with support from start-up funds from the MUSC COM and an award from SC Epscor to GH. The content is solely the responsibility of the authors and does not necessarily represent the official views of the Medical University of South Carolina.

\section{References}

1. Alberts B, Johnson A, Lewis J et al (2015) Analyzing cells, molecules, and systems. In: Molecular biology of the cell, 6 th edn. Garland Science, USA, pp 440-528

397 2. Garrett CT (2015) Molecular biology basics inthe "Omics" Era: genes to proteins. In: Idowu OM, 398 Dumur IC, Garrett TC (eds) Molecular oncology testing for solid tumors: a pragmatic approach. 399 Springer International Publishing, Cham, pp 3-65

400 3. Wang Z, Gerstein M, Snyder M (2009) RNA-Seq: a revolutionary tool for transcriptomics. Nat Rev 401 Genet 10(1):57-63

402 4. Mayer-Scho"nberger V, Cukier K (2013) Big data: a revolution that will transform how we live, 403 work, and think. Harcourt, Houghton Mifflin

404 5. Sengupta S, Bolin JM, Ruotti V et al (2011) Single read and paired end mRNA-Seq Illumina libraries 405 from 10 nanograms total RNA. J Vis Exp (56):e3340. https://doi.org/10.3791/3340

406 6. Cock PJ, Fields CJ, Goto N et al (2010) The Sanger FASTQ file format for sequences with quality 407 scores, and the Solexa/Illumina FASTQ variants. Nucleic Acids Res 38 (6):1767-1771 
408 7. Illumina (2011) Quality scores for nest generation sequencing: assessing sequencing accuracy

409 using phred quality scoring.

410 https://www.illumina.com/Documents/products/technotes/technote_Q-Scores.pdf

411 8. Zhang T, Guo L, Creighton CJ et al (2016) A genetic cell context-dependent role for ZEB1 in lung

412 cancer. Nat Commun 7:12231

413 9. Lenferink AE (2017) Epithelial-to-Mesenchymal transition (EMT): the good, the bad, and the ugly.

414 In: Wang E (ed) Cancer systems biology. CRC Press, Florida

415 10. Andrews S (2010) FastQC: a quality control tool for high throughput sequence data.

416 http://www.bioinformatics.babraham.ac.uk/ projects/fastqc

417 11. Gordon A, Hannon G (2010). Fastx-Toolkit. In: FASTQ/A short-reads preprocessing tools.

418 http://hannonlab.cshl.edu/fastx_toolkit/

419 12. Martin M (2011) Cutadapt removes adapter sequences from high-throughput sequencing reads.

420 EMBnetjournal 17(1):10-12

421 13. Speir ML, Zweig AS, Rosenbloom KR et al (2016) The UCSC genome browser database: 2016

422 update. Nucleic Acids Res 44(D1): D717-D725

423 14. Trapnell C, Roberts A, Goff $L$ et al (2012) Differential gene and transcript expression analysis of

424 RNA-seq experiments with TopHat and cufflinks. Nat Protoc 7(3):562-578

425 15. Trapnell C, Pachter L, Salzberg SL (2009) TopHat: discovering splice junctions with RNA-Seq.

426 Bioinformatics 25(9):1105-1111

427 16. Soneson C, Delorenzi M (2013) A comparison of methods for differential expression analysis of 428 RNA-seq data. BMC Bioinformatics 14:91

429 17. Love MI, Huber W, Anders S (2014) Moderated estimation of fold change and dispersion for RNA430 seq data with DESeq2. Genome Biol 15(12):550

431 18. Law CW, Chen $Y$, Shi $W$ et al (2014) Voom: precision weights unlock linear model analysis tools 432 for RNA-seq read counts. Genome Biol 15(2):R29

433 19. Smyth GK (2005) Limma: linear models for microarray data. In: Gentleman R, Carey VJ, Huber W, 434 Irizarry RA, Dudoit S (eds) Bioinformatics and computational biology solutions using R and 435 bioconductor. Statistics for biology and health. Springer, New York, NY

436 20. Anders S, Pyl PT, Huber W (2015) HTSeq-a python framework to work with highthroughput 437 sequencing data. Bioinformatics 31(2):166-169

438 21. Trapnell C, Williams BA, Pertea G et al (2010) Transcript assembly and quantification by RNA-Seq 439 reveals unannotated transcripts and isoform switching during cell differentiation. Nat Biotechnol $440 \quad 28(5): 511-515$

441 22. Conesa A, Madrigal P, Tarazona S (2016) A survey of best practices for RNA-seq data analysis. 442 Genome Biol 17(1):181

443 23. Bourgon R, Gentleman R, Huber W (2010) Independent filtering increases detection power for 444 high-throughput experiments. Proc Natl Acad Sci U S A 107(21):9546-9551 
446 Genome Biol 15(12):550

447 25. Ritchie ME, Phipson B, Wu D et al (2015) Limma powers differential expression analyses for RNA-

448 sequencing and microarray studies. Nucleic Acids Res 43(7):e47

449 26. Datta S, Nettleton D (2014) Statistical analysis of next generation sequencing data. In: Datta S,

450 Nettleton D (eds) Frontiers in probability and the statistical sciences. Springer International

451 Publishing, Switzerland, pp 1-32

452 27. Hulsen T, De Vlieg J, Alkema W (2008) BioVenn-a web application for the comparison and

453 visualization of biological lists using areaproportional Venn diagrams. BMC Genomics 9:488

454 28. Stelzer G, Dalah I, Stein TI et al (2011) In-silico human genomics with GeneCards. Hum Genomics 455 5(6):709-717

456 29. Uhlen $M$, Oksvold P, Fagerberg L (2010) Towards a knowledge-based human protein atlas. Nat

457 Biotechnol 28(12):1248-1250

458 30. Subramanian A, Tamayo P, Mootha VK et al (2005) Gene set enrichment analysis: a knowledge-

459 based approach for interpreting genome-wide expression profiles. Proc Natl Acad Sci U S A

460 102(43):15545-15550

461 31. Chen J, Bardes EE, Aronow BJ et al (2009) ToppGene suite for gene list enrichment analysis and 462 candidate gene prioritization. Nucleic Acids Res 37(Web Server): W305-W311

463 32. Mootha VK, Lindgren CM, Eriksson KF et al (2003) PGC-1alpha-responsive genes involved in 464 oxidative phosphorylation are coordinately downregulated in human diabetes. Nat Genet 34(3):267$465 \quad 273$

466 33. Gene Set Enrichment Analysis (GSEA) User Guide (2010), http://software.broadinstitute.

$467 \mathrm{org} / \mathrm{gsea} / \mathrm{doc} / \mathrm{GSEAU}$ serGuideFrame.html

468 34. Wold B, Myers RM (2008) Sequence census methods for functional genomics. Nat Methods 469 5(1):19-21

470 35. Alon U (2006) An introduction to systems biology: design principles of biological circuits. CRC

471 Press, Florida

472

473 


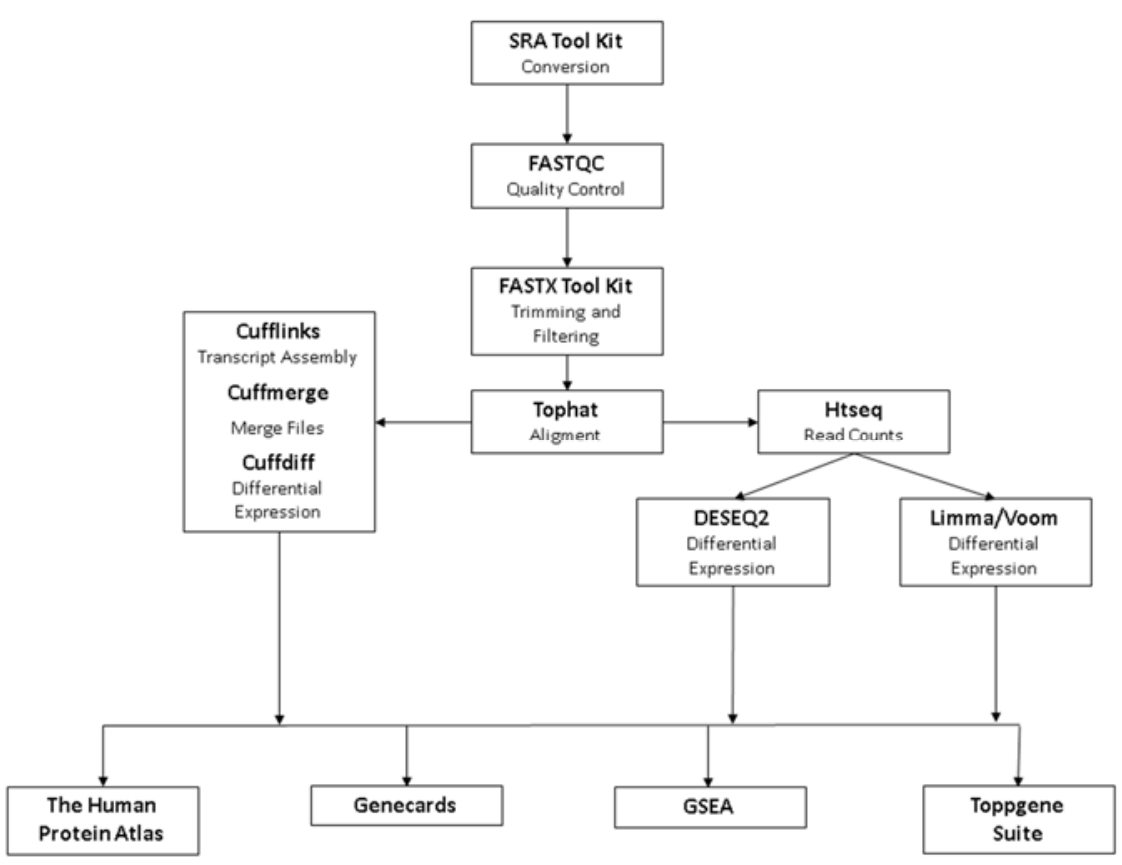

481

Fig. 1 Flowchart summarizing analyses from RNA seq data. The conversion step with SRA tool kit is 483 generally only used with data from repositories

484

485

486

487

488

489

490

491

492

493

494

495

496 
A

QSRR3478269.1 1 length $=102$

GGA.AATTTAAAAAAATACACATGGCCAGGCCCCAGCCCAAATCACTAATAACCCGGAGCATATGCTGTTTGGTCTCAGTAGGCTCCTAAATATGGGATTCCT +SRR3478269.1 1 length=102

CCCFFFFFHFFHHJIIJJJIIIJJIJJJJIIJJJJJJJJIJIJIJJGJJJJCCCFFDFFHHDHFGIGIIJJJIIJIFHGHIJIJGGIEHJJFIJIJIIIJJJ

B

497

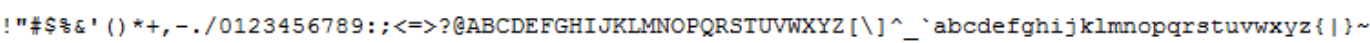

498 Fig. 2 FASTQ Example. Sample SRR3478269 from Dataset GSM2144086. (a) First 4 lines of the FASTQ

499 file of the Sample SRR3478269 from Dataset GSM2144086. (b) FASTQ quality score in ascending

500 order from the left to right

501

502

503

504

505

506

507

508

509

510

511

512 


\section{Good Report}

\section{EFastQC Report}
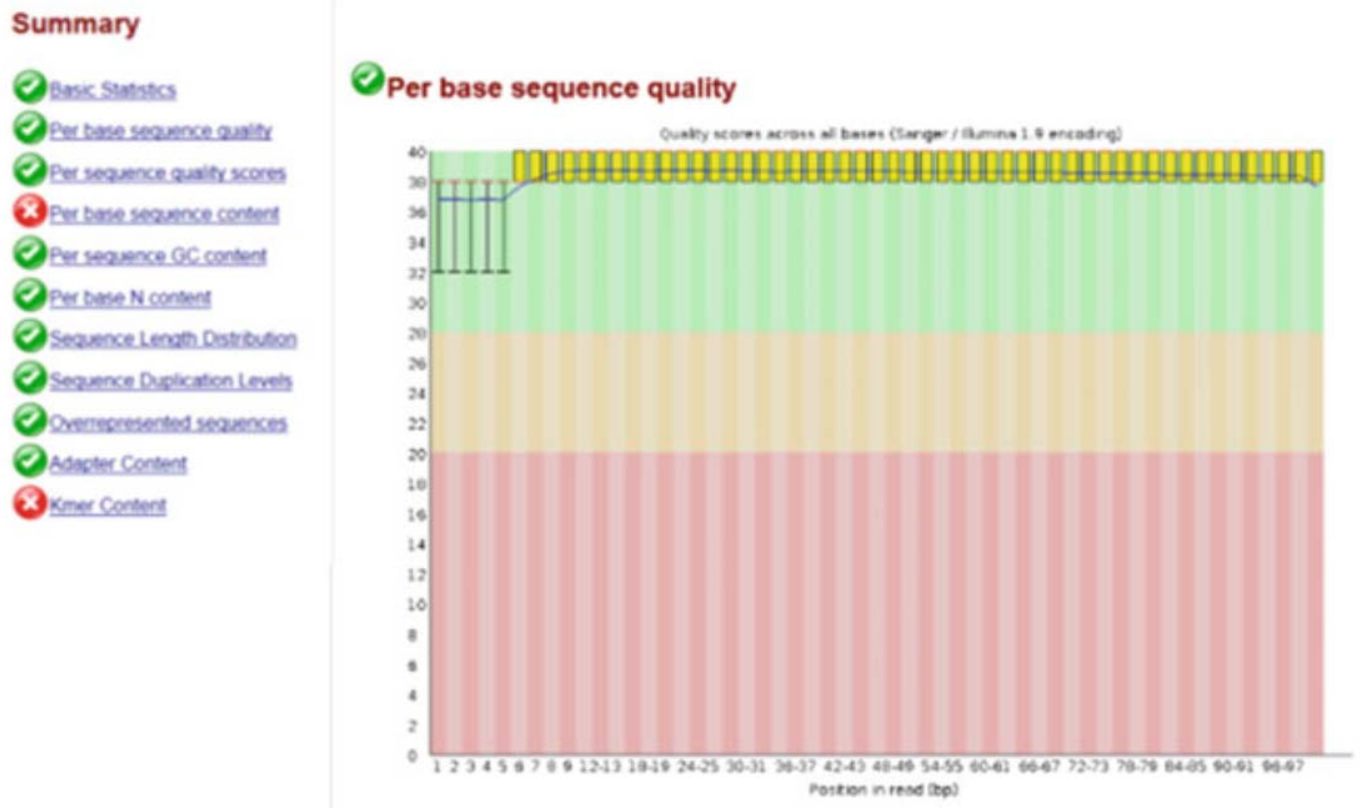

\section{Bad Report}

\section{EFastQC Report}

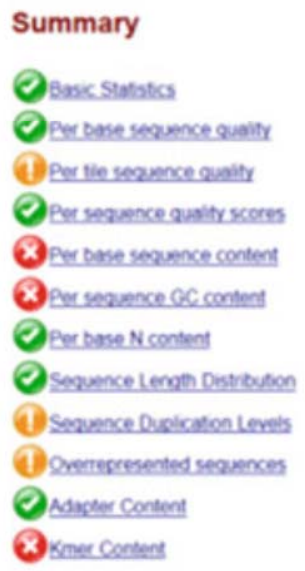

Per base sequence quality

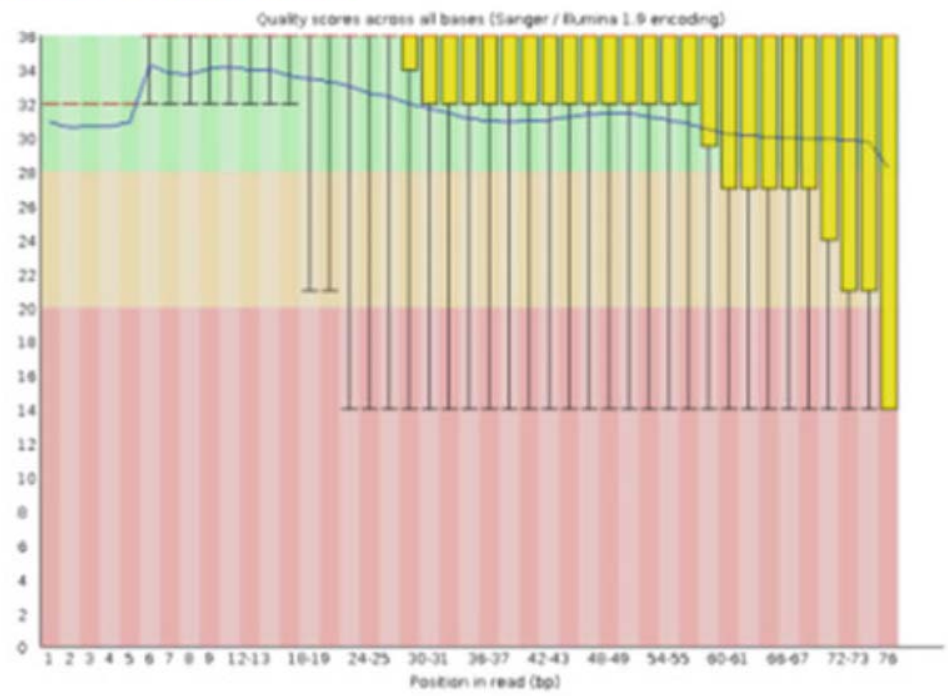

Fig. 3 FastQC report. Example FastQC report for a good quality sample (Upper Panel) and a poor 


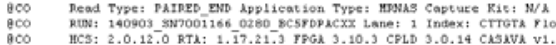
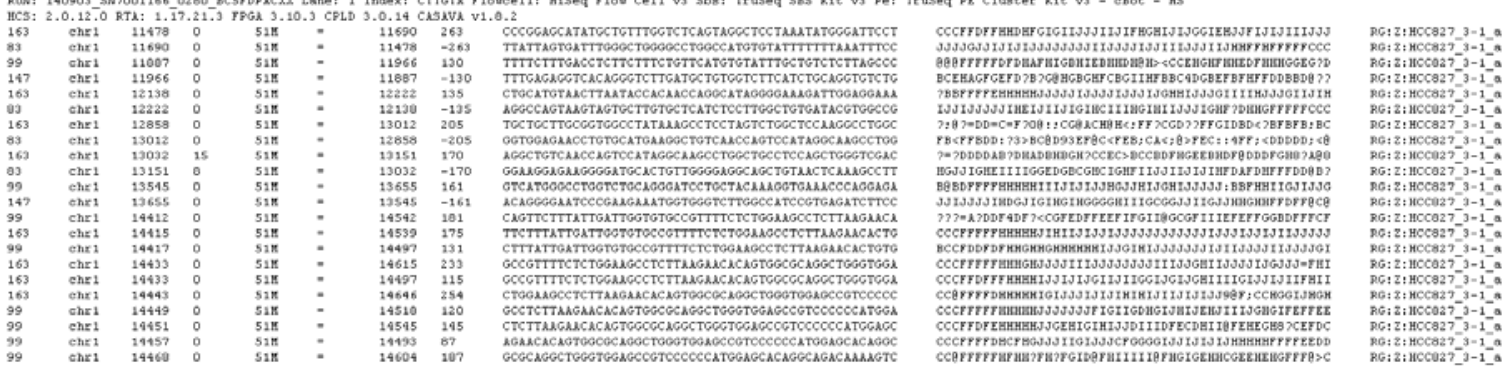

518 Fig. 4 Example SAM file. First several lines of the SAM file from Sample SRR3478269 from Dataset 519 GSM2144086.

520

521

522

523

524

525

526

527

528

529

530

531

532

533

534

535 


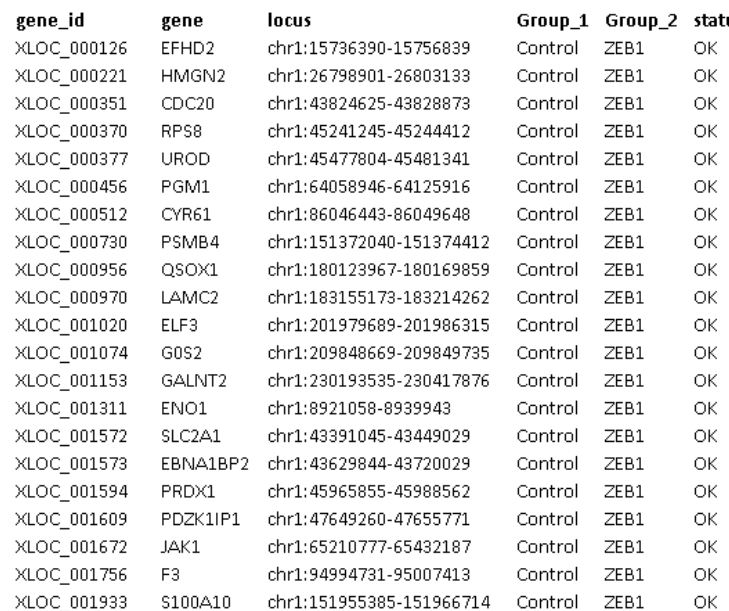

value_1 value_2 $\log 2$ (fold_change) test_stat p_value q_value $\begin{array}{lllllll}202.415 & 95.5723 & -1.08265 & -2.95750 & 5.00 E-05 & 0.00051\end{array}$ $\begin{array}{llllll}161.569 & 235.038 & 0.540743 & 1.63467 & 0.0131 & 0.0473428\end{array}$ $\begin{array}{lllllll}225.636 & 318.984 & 0.499487 & 1.62614 & 0.0133 & 0.0478431\end{array}$ $\begin{array}{lllllll}2517.32 & 1969.36 & -0.354161 & -1.67685 & 0.01245 & 0.045781\end{array}$ $\begin{array}{llllll}71.9628 & 190.767 & 1.32881 & 1.99676 & 0.0012 & 0.0079017\end{array}$ $\begin{array}{lllllll}142.789 & 88.7964 & -0.685316 & -1.38816 & 0.00725 & 0.0320071\end{array}$ $\begin{array}{lllllll}701.992 & 521.97 & -0.427489 & -1.96439 & 0.0023 & 0.0135386\end{array}$ $\begin{array}{lllllll}254.692 & 435.399 & 0.772454 & 2.2223 & 0.00425 & 0.0207689\end{array}$ $\begin{array}{llllll}264.486 & 123.342 & -1.10053 & -2.63871 & 5.00 \mathrm{E}-05 & 0.000518\end{array}$ $\begin{array}{llllll}177.744 & 13.4283 & -3.72645 & -4.96459 & 5.00 E-05 & 0.000518\end{array}$ $\begin{array}{llllll}123.944 & 20.7934 & -2.10589 & -3.44096 & 5.00 E-05 & 0.000518\end{array}$ $\begin{array}{lllllll}291.39 & 16.4758 & -4.14453 & -2.88957 & 0.00595 & 0.0278503\end{array}$ $\begin{array}{lllllll}99.1635 & 167.39 & 0.755334 & 1.55569 & 0.00275 & 0.0147362\end{array}$ $\begin{array}{lllllll}2012.97 & 2502.59 & 0.314096 & 1.46414 & 0.01065 & 0.0417349\end{array}$ $\begin{array}{llllll}281.788 & 139.861 & -1.01062 & -3.46746 & 5.00 E-05 & 0.00051\end{array}$ $\begin{array}{lllllll}124.382 & 212.616 & 0.773473 & 1.53116 & 0.0117 & 0.0437062\end{array}$ $\begin{array}{lllllll}528.434 & 802.64 & 0.60303 & 1.88551 & 0.0008 & 0.006035\end{array}$ $\begin{array}{lllllll}358.718 & 37.0944 & -3.27358 & -3.42087 & 0.0076 & 0.0326067\end{array}$ $\begin{array}{rrrrrr}358.718 & 37.0944 & -3.27358 & -3.42087 & 0.0076 & 0.0328067 \\ 116.975 & 62.8851 & -0.895414 & -2.55947 & 5.00 \mathrm{E}-105 & 0.000518\end{array}$ $\begin{array}{llllll}295.327 & 72.2413 & -2.03142 & -4.48758 & 5.00 \mathrm{E}-05 & 0.000518\end{array}$ $\begin{array}{llllll}337.267 & 168.961 & -0.997202 & -2.53059 & 0.0017 & 0.0104839\end{array}$

537 Fig. 5 Cuffdiff output. Top 20 differentially expressed genes from Cuffdiff analysis

538 


$\begin{array}{lc}\text { Symbol } & \text { counts } \\ \text { A1BG-AS1 } & 1 \\ \text { AAAS } & 3 \\ \text { AACS } & 2 \\ \text { AAGAB } & 5 \\ \text { AAK1 } & 1 \\ \text { AAMP } & 4 \\ \text { AAR2 } & 1 \\ \text { AARS } & 5 \\ \text { AASS } & 1 \\ \text { AATF } & 3 \\ \text { ABCA1 } & 10 \\ \text { AEL2 } & 11 \\ \text { ACTG1 } & 205 \\ \text { CEP41 } & 1 \\ \text { EEF1A1 } & 419 \\ \text { GAFDH } & 453 \\ \text { TTC3 } & 12 \\ \text { TTH3 } & 12 \\ \text { TUBA1B } & 200 \\ \text { TUBA1C } & 202\end{array}$

545

546 Fig. 6 HTSeq output. Example of count results from sample GSM2144086

547

548

549

550

551

552

553

554

555 
basemean log2Foldchange IffSE stat pvalue padj $\begin{array}{lllllll}46.51829 & -2.440349331 & 0.27796973 & -8.77919 & 1.655 .18 & 4.0255 .15\end{array}$ $\begin{array}{llllllll}155.1586327 & -1.136943225 & 0.13453789 & -8.75073 & 2895-17 & 4.715-14\end{array}$ $\begin{array}{lllllll}74,02538642 & 1.783708582 & 0.21425014 & 8.325355 & 8.41 E-17 & 1.03 E-13\end{array}$ $\begin{array}{llllllll}25.99212881 & -3.852052972 & 0.46562369 & -8.27289 & 1.31 E-16 & 1.29 E-13\end{array}$

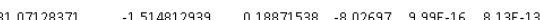
$\begin{array}{lllllll}21.87722077 & -4.679926755 & 0.60298745 & -7.76123 & 8.41515 & 5.875 .12\end{array}$ $\begin{array}{lllllll}20.97514876 & -4.914503581 & 0.63920592 & -7.768845 & 1.495-14 & 9.095-12\end{array}$ $\begin{array}{lllllll}20.96486972 & -3.393701917 & 0.46634748 & -7.2772 & 3.41 E \cdot 13 & 1.66 E-10\end{array}$ $\begin{array}{lllllll}22.22526049 & -3.350144599 & 0.43211452 & -7.29007 & 3.105-13 & 1.665-10\end{array}$ $\begin{array}{llllllll}17.94658771 & -4.17558093 & 0.59055798 & -7.79236 & 6375-13 & 2.835-10\end{array}$ $\begin{array}{llllllll}35.42369692 & 2.206368912 & 0.30934126 & 7.732475 & 9.86 E-13 & 4015 \cdot 10\end{array}$ $\begin{array}{llllllll}16.59529808 & -4506994063 & 0.65133486 & -705765 & 1695-12 & 63365-10\end{array}$ $\begin{array}{lllllllll}94.06276097 & -1.152540615 & 0.1702325 & -6.77039 & 1.28 E-11 & 4.49 E-09\end{array}$ $\begin{array}{lllllll}13.64243193 & -5.136508256 & 0.76190433 & -6.7418 & 1.56 E-11 & 5.09 E-09\end{array}$ $\begin{array}{lllllll}13.4714644 & -4.695365975 & 0.70570744 & -6.69925 & 3.15 E-11 & 9.62 E-09\end{array}$ $\begin{array}{lllllll}63.29305063 & -1.287364914 & 0.20737209 & -6.208 & 5.37 E-10 & 1.54 E-07\end{array}$ $\begin{array}{llllll}31.04926848 & -1.936850937 & 0.31515463 & -6.14572 & 7.96 E-10 & 2.16 E-07\end{array}$ $\begin{array}{llllll}9.925612632 & -4.723189071 & 0.79409378 & -6.02375 & 1.70 \mathrm{E}-09 & 4.38 \mathrm{E}-07\end{array}$

557 Fig. 7 DESeq2 output. Top 20 differentially expressed genes from DESeq2 analyzes 


\begin{tabular}{|c|c|c|c|c|c|c|}
\hline & & & & & & \\
\hline LCP & 85418 & 6.573509 & -16.9375 & $2.28 \mathrm{E}-07$ & & 12577 \\
\hline FAM & 33212 & 812479 & 217 & -06 & & 455 \\
\hline ESRP1 & & & & & & \\
\hline$\varnothing 0$ & & & & & & 6808 \\
\hline COL4 & & & & & & 180 \\
\hline & & & & & & 1587 \\
\hline COL4 & .67745 & 5143 & -11.419 & -06 & & 8877 \\
\hline $\mathrm{LAM}$ & & & & & & \\
\hline & & & & & & \\
\hline $\mathrm{LCN}$ & .38404 & 5079 & & 06 & & 573 \\
\hline LFRO & & & & & & \\
\hline NF5 & 91 & & & & & 181 \\
\hline CGN & .58095 & 5.4 & & -06 & 222 & 0012 \\
\hline $\mathrm{ECH}$ & & & & & & \\
\hline SAA1 & .31258 & 6.831029 & -10.2951 & $9.04 \mathrm{E}-06$ & 822 & 3766 \\
\hline GALNNT3 & -3.40411 & 5.348473 & -10.263 & $9.24 \mathrm{E}-06$ & & 3441 \\
\hline SCNG & & 5.396724 & & & & 73631 \\
\hline CDA & -3.70996 & 5.501396 & -9.97544 & $1.13 \mathrm{E}-05$ & 0.003 & 3.967801 \\
\hline NFFSF1DA & -3.28325 & 5.288044 & -9.67495 & 1. 41E-05 & 0.003625 & 3.766785 \\
\hline SPTLC3 & & 5.575972 & & & & .72187 \\
\hline $\mathrm{CDH} 3$ & -3.45332 & 7.44935 & -9.45097 & $1.67 \mathrm{E}-0.5$ & 0.00386 & 3.612084 \\
\hline
\end{tabular}

566

567 Fig. 8 Limma/Voom output. Top 20 differentially expressed genes from Limma/Voom analyses 


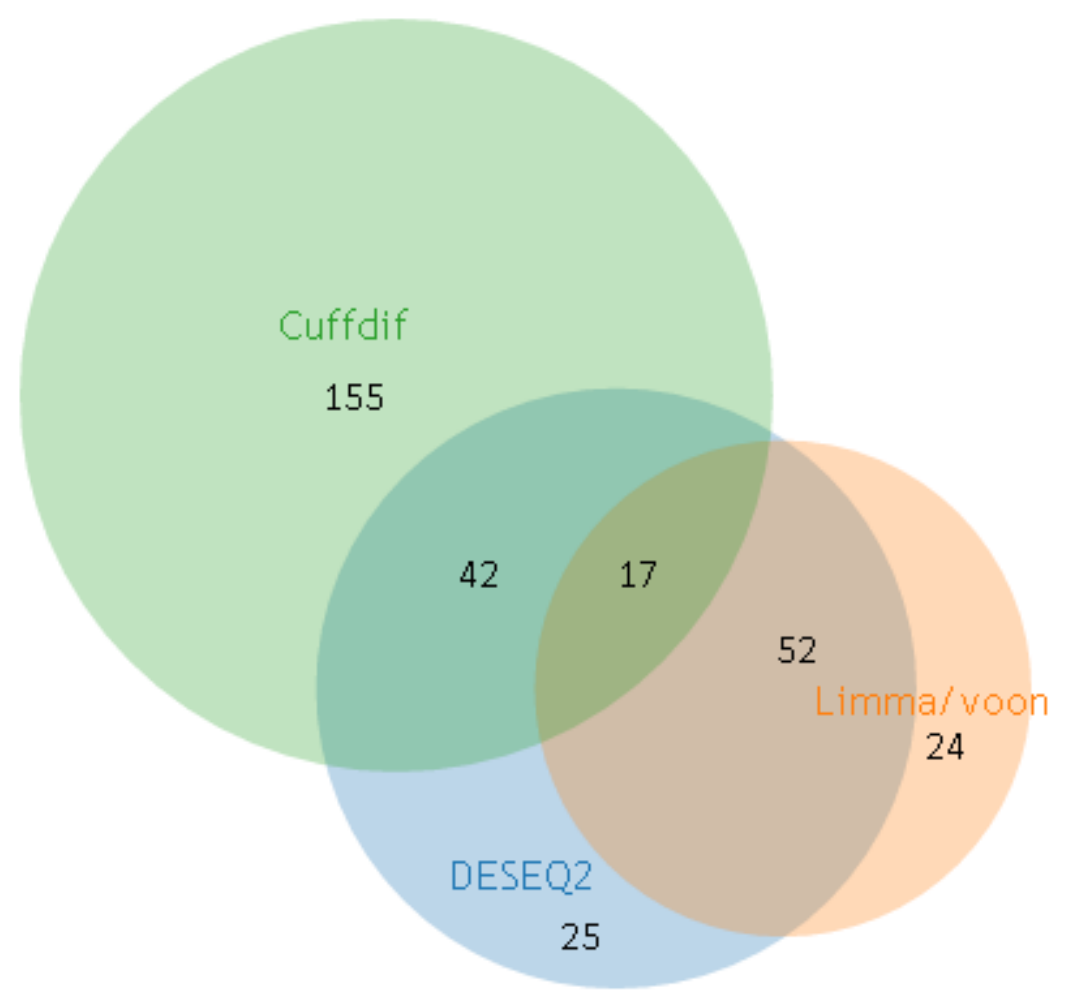

569

570 Fig. 9 Venny output. Proportional Venn diagram comparing the list of differentially expressed genes 571 uncovered by Cuffdiff, DESeq2 and Limma/Voom, where differential expression was determined at 572 adjusted $p$-value of $<0.05$ for all the three methods

573

574 


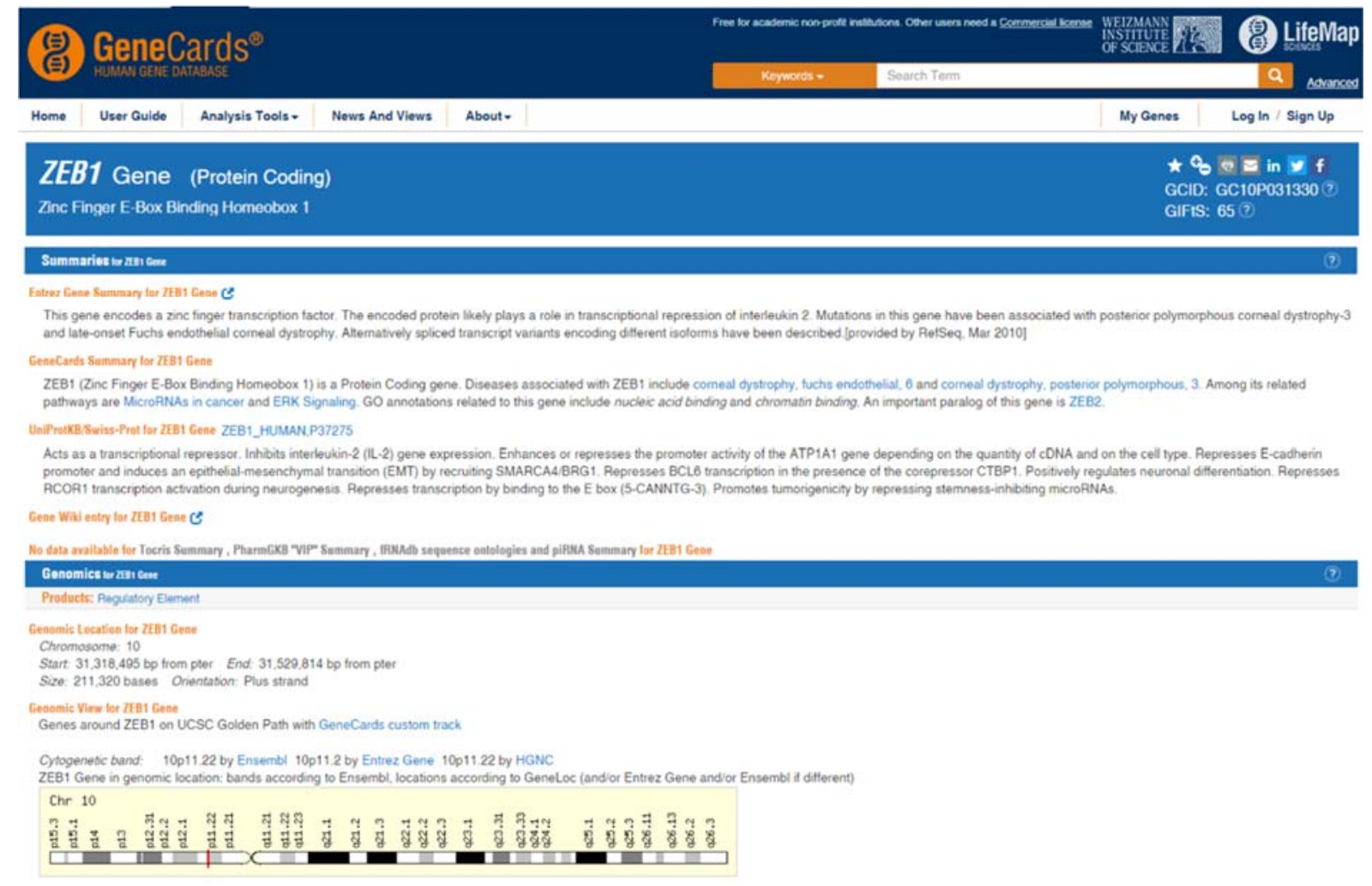

576 Fig. 10 ZEB1 edited description in Genecards

577 


\section{THE HUMAN PROTEIN ATLAS ${ }^{\circ}$}

ZEB1
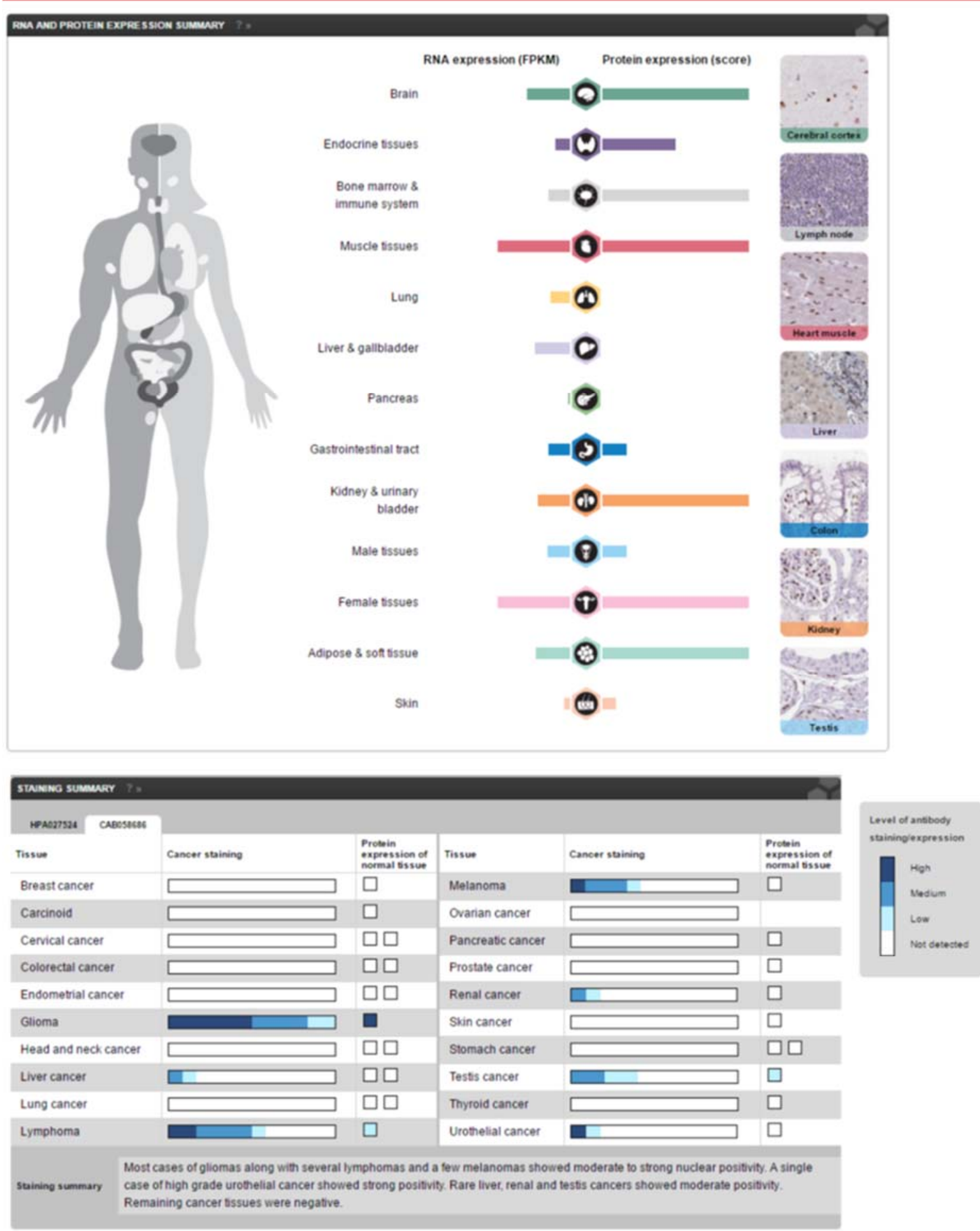

Fig. 11 ZEB1 edited description in The Human Protein Atlas website 

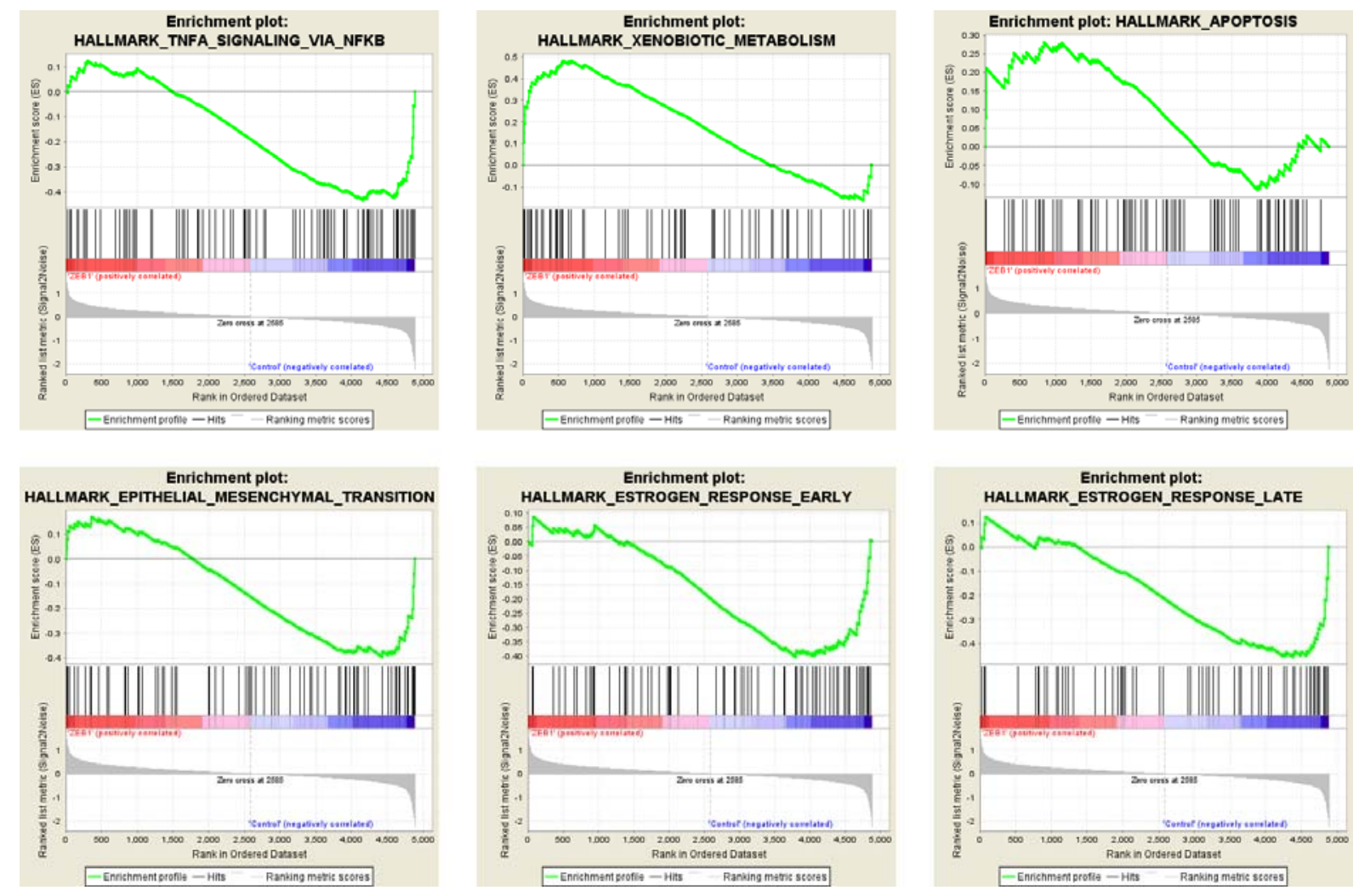

Fig. 12 Results from GSEA desktop version (using the transformed table from Limma/Voom as input) 


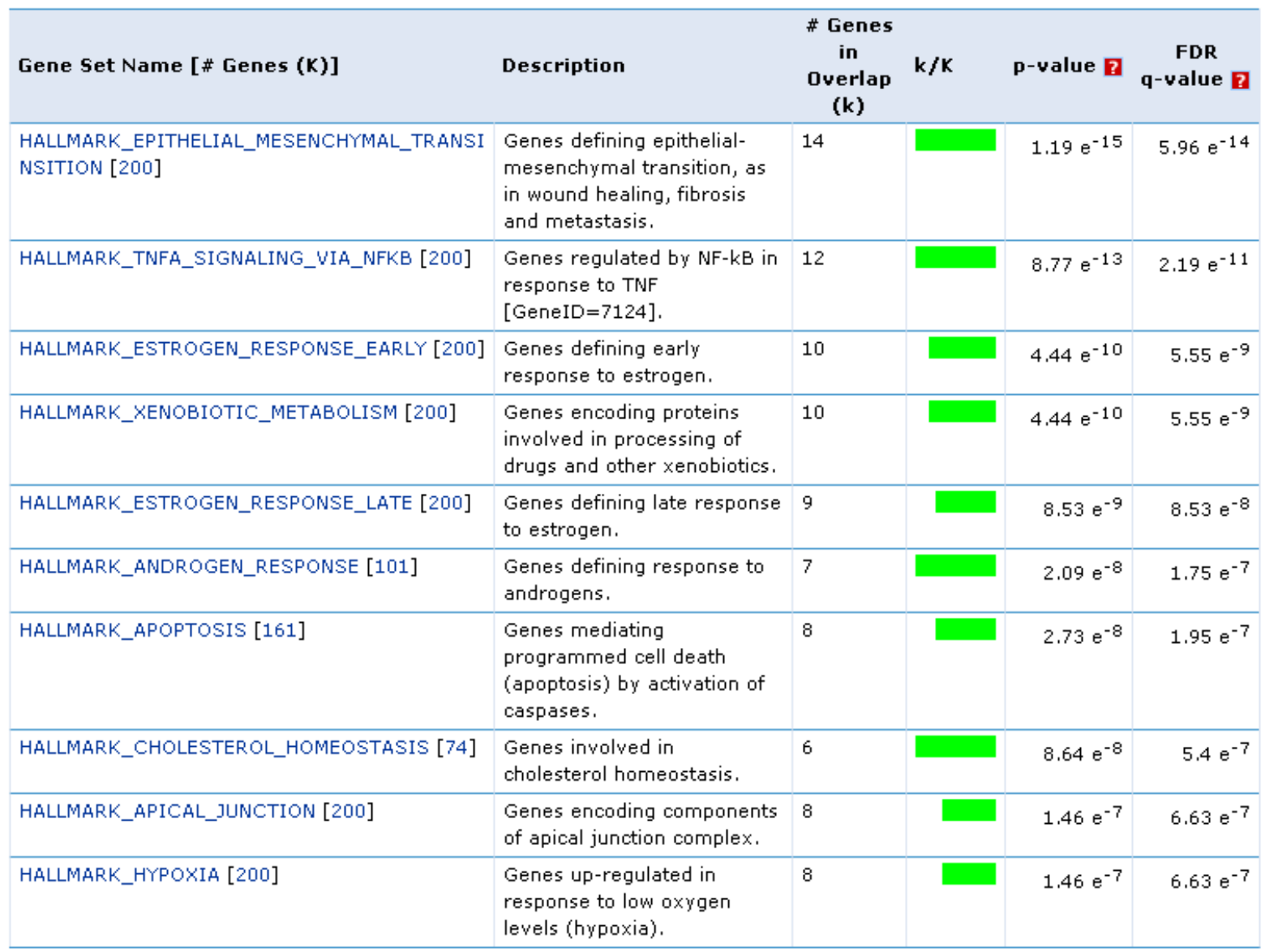

Fig. 13 GSEA web tool Analysis. Top 10 Hallmarks gene sets enriched for the DE gene list obtained 587 from DESeq2 
1: G0: Molecular Function [Display Chart] 557 annotations before applied cutoff / 18661 genes in category

\begin{tabular}{|l|l|l|l|l|l|l|r|r|r|r|}
\hline ID & Name & Source & pValue & FDR B\&H & FDR B\&Y & Bonferroni & Genes from Input & Genes in Annotation \\
\hline 1 & GO:0005198 & structural molecule activity & $8.892 \mathrm{E}-8$ & $4.953 \mathrm{E}-5$ & $3.418 \mathrm{E}-4$ & $4.953 \mathrm{E}-5$ & 21 & 762 \\
\hline 2 & GO:0008201 & heparin binding & $2.937 \mathrm{E}-6$ & $8.181 \mathrm{E}-4$ & $5.645 \mathrm{E}-3$ & $1.636 \mathrm{E}-3$ & 9 & 167 \\
\hline 3 & GO:0045236 & CXCR chemokine receptor binding & $5.301 \mathrm{E}-6$ & $9.841 \mathrm{E}-4$ & $6.791 \mathrm{E}-3$ & $2.952 \mathrm{E}-3$ & 4 & 17 \\
\hline 4 & GO:0005200 & structural constituent of cytoskeleton & $1.312 \mathrm{E}-5$ & $1.827 \mathrm{E}-3$ & $1.261 \mathrm{E}-2$ & $7.310 \mathrm{E}-3$ & 7 & 110 \\
\hline 5 & GO:0005539 & glycosaminoglycan binding & $2.609 \mathrm{E}-5$ & $2.906 \mathrm{E}-3$ & $2.006 \mathrm{E}-2$ & $1.453 \mathrm{E}-2$ & 9 & 219 \\
\hline
\end{tabular}

\section{Show 22 more annotations}

2: G0: Biological Process [Display Chart] 3331 annotations before applied cutoff / 18623 genes in category

\begin{tabular}{|c|c|c|c|c|c|c|c|c|c|}
\hline & ID & Name & Source & pvalue & FDR $\mathrm{B} \& \mathrm{H}$ & FDR B\&Y & Bonferroni & Genes from Input & Genes in Annotation \\
\hline 1 & GO:0060429 & epithelium development & & 2.597E-10 & $8.652 \mathrm{E}-7$ & $7.517 \mathrm{E}-6$ & $8.652 \mathrm{E}-7$ & 32 & 1296 \\
\hline 2 & GO:0042127 & regulation of cell proliferation & & $5.978 \mathrm{E}-10$ & $9.956 \mathrm{E}-7$ & $8.650 \mathrm{E}-6$ & $1.991 \mathrm{E}-6$ & 36 & 1666 \\
\hline 3 & GO:0040012 & regulation of locomotion & & $1.500 \mathrm{E}-9$ & $1.500 \mathrm{E}-6$ & $1.303 E-5$ & $4.996 \mathrm{E}-6$ & 25 & 866 \\
\hline 4 & GO:0040011 & locomotion & & $1.801 \mathrm{E}-9$ & $1.500 \mathrm{E}-6$ & $1.303 \mathrm{E}-5$ & $5.999 \mathrm{E}-6$ & 36 & 1735 \\
\hline 5 & GO:0048870 & cell motility & & 2.897E-9 & $1.609 \mathrm{E}-6$ & $1.398 \mathrm{E}-5$ & $9.651 \mathrm{E}-6$ & 32 & 1428 \\
\hline
\end{tabular}

\section{Show 45 more annotations}

3: G0: Cellular Component [Display Chart] 360 annotations before applied cutoff / 19061 genes in category

\begin{tabular}{|l|l|l|l|l|l|r|r|r|r|}
\hline ID & Name & Source & pValue & FDR B\&H & FDR B\&Y & Bonferroni & Genes from Input Genes in Annotation \\
\hline 1 & GO:0005925 & focal adhesion & & $3.882 \mathrm{E}-12$ & $7.361 \mathrm{E}-10$ & $4.758 \mathrm{E}-9$ & $1.397 \mathrm{E}-9$ & 20 & 393 \\
\hline 2 & GO:0005924 & cell-substrate adherens junction & $4.888 \mathrm{E}-12$ & $7.361 \mathrm{E}-10$ & $4.758 \mathrm{E}-9$ & $1.760 \mathrm{E}-9$ & 20 & 398 \\
\hline 3 & GO:0030055 & cell-substrate junction & $6.134 \mathrm{E}-12$ & $7.361 \mathrm{E}-10$ & $4.758 \mathrm{E}-9$ & $2.208 \mathrm{E}-9$ & 20 & 403 \\
\hline 4 & GO:0005912 & adherens junction & $2.181 \mathrm{E}-11$ & $1.963 \mathrm{E}-9$ & $1.269 \mathrm{E}-8$ & $7.853 \mathrm{E}-9$ & 21 & 484 \\
\hline 5 & GO:0070161 & anchoring junction & $4.466 \mathrm{E}-11$ & $3.216 \mathrm{E}-9$ & $2.079 \mathrm{E}-8$ & $1.608 \mathrm{E}-8$ & 21 & 503 \\
\hline
\end{tabular}

590 Fig. 14 Results from TOPPFUN. Gene Ontology terms enriched for the gene list obtained from 